\title{
Geodetic Precession in PSR B1534+12
}

\section{H. Stairs}

University of Manchester, Jodrell Bank Observatory, Macclesfield, Cheshire SK11 9DL UK

S. E. Thorsett

Department of Astronomy and Astrophysics, University of California, 1156 High St., Santa Cruz, CA 95064 USA

J. H. Taylor

Joseph Henry Laboratories and Physics Department, Princeton University, Princeton, NJ 08544 USA

\section{Z. Arzoumanian}

NASA Goddard Space Flight Center, Mailstop 662.0, Greenbelt, MD 20771 USA

\begin{abstract}
We present Arecibo observations of PSR B1534+12 which confirm previous suggestions that the pulse profile is evolving secularly. This effect is similar to that seen in PSR B1913+16, and is almost certainly due to general relativistic precession of the pulsar's spin axis.
\end{abstract}

\section{Introduction}

Binary pulsars in close, highly eccentric orbits have long provided the best strong-field tests of the predictions of gravitational theories. Timing observations of PSR B1913+16 have allowed the measurement of three "post-Keplerian" parameters: the rate of periastron advance, $\dot{\omega}$, the time-dilation and gravitationalredshift parameter, $\gamma$, and the rate of orbital period decay, $\dot{P}_{b}$ (Taylor \& Weisberg 1989); while PSR B1534+12 permits, in addition, the measurement of the Shapiro-delay parameters, $r$ and $s$ (Stairs et al. 1998). These observations have yielded highly precise tests of both the radiative and quasi-static predictions of the theory of general relativity.

General relativity also predicts geodetic precession. The pulsar spin axis, if misaligned with the orbital angular momentum vector, will evolve according to:

$$
\frac{d \mathrm{~S}_{1}}{d t}=\Omega_{1}^{\mathrm{spin}} \times \mathrm{S}_{1}
$$

where, in general relativity,

$$
\Omega_{1}^{\mathrm{spin}}=\frac{G^{3 / 2} M^{1 / 2} \mu}{c^{2} a^{5 / 2}\left(1-e^{2}\right)}\left[2+\frac{3}{2} \frac{m_{2}}{m_{1}}\right]
$$




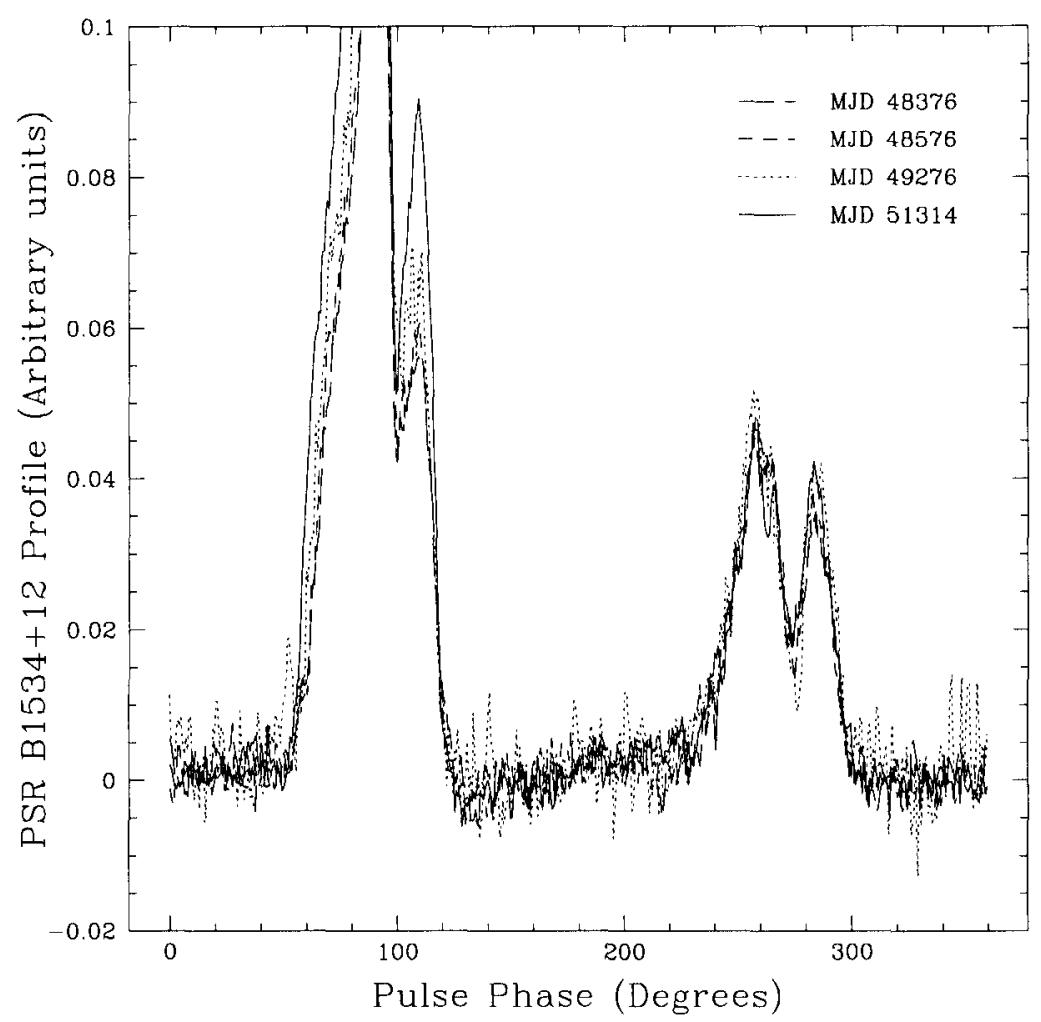

Figure 1. Evolution of the $1400 \mathrm{MHz}$ pulse profile of PSR B1534+12 with time, using the interpulse as a fiducial reference.

where $G$ is Newton's constant, $M$ is the total mass of the system, $\mu$ the reduced mass, $c$ the speed of light, $a$ the projected semi-major axis of the orbit, $e$ the orbital eccentricity, and $m_{1}$ and $m_{2}$ the pulsar and companion masses respectively. For PSR $\mathrm{B} 1534+12, \Omega_{1}^{\text {spin }}$ amounts to $0.52^{\circ} \mathrm{yr}^{-1}$; an entire precession cycle would take approximately 690 years. As the angle between the spin axis and the line-of-sight to the pulsar changes, the observed cut across the pulsar emission region will also change, and result in the secular evolution of the pulse profile. The long-term change in profile shape of PSR B1913+16 has been interpreted as evidence for this precession (e.g, Weisberg, Romani \& Taylor 1989; Kramer 1998; Weisberg \& Taylor, these proceedings). Similar profile evolution in PSR B1534+12 was noted by Arzoumanian (1995) in examining Arecibo observations from 1990-94; these changes took the form of an apparent increase with time in the strength of the interpulse relative to the main pulse at $1400 \mathrm{MHz}$. 




Figure 2. The $1400 \mathrm{MHz}$ pulse profile of PSR B1534+12. The solid, dashed and dotted lines indicate total, linear and circular power, respectively. The polarization position angle is shown in the lower panel.

\section{Observations and Results}

In May 1999, we carried out $1400 \mathrm{MHz}$ observations of PSR B1534+12 at Arecibo Observatory to investigate whether this secular evolution was still occurring. During the Arecibo upgrade period, we had developed a $10-\mathrm{MHz}$ bandwidth coherent-dedispersion baseband recorder, known as "Mark IV," which we used in parallel with the older $2 \times 32 \times 1.25 \mathrm{MHz}$ "Mark III" filterbank. This strategy enabled us to compare our newest profile with those of Arzoumanian, which were also obtained with Mark III, to identify any possible small instrumental differences between the two observing systems, and to establish a baseline coherently-dedispersed profile against which to compare future observations.

Our recent data confirm Arzoumanian's observations of precession, and reveal new detail in the pulse profile evolution. This can be seen in Figure 1, in 
which the small interpulse is taken as a reference point. The profile dated MJD 51314 is from our May 1999 observations with the Mark III system; the earlier three profiles are taken from Arzoumanian (1995). It is clear that the "wings" of the main pulse are growing relative to the interpulse, and it appears that the total width at roughly the half-height of the wings is also increasing with time. The peak height of the main pulse relative to the interpulse is also changing, though not monotonically: it decreased between MJDs 48376 and 49276, then began increasing again at some point prior to MJD 51314.

While the change in relative strength of the profile components is certainly an indication that we are viewing a slightly different part of the emission region, this cannot by itself provide an estimate of the change in viewing angle. However, if the apparent change in width of the base of the main pulse is real, it will be possible to combine this information with the magnetic geometry determined from the Rotating Vector Model (RVM) to put together a model of the beam shape and change in viewing angle, as has been done for PSR B1913+16 (e.g., Cordes, Wasserman \& Blaskiewicz 1990, Kramer 1998, Kasterogiou et al., these proceedings), and perhaps even to test quantitatively the rate at which precession is occurring. The latter may be possible for PSR B1534+12 because its magnetic geometry is very well constrained from polarization observations. With the Mark IV instrument, we have obtained a high-resolution polarization profile of this pulsar at $1400 \mathrm{MHz}$ (see Figure 2); the resulting fit to the RVM agrees fairly well with the "Fit B" fit derived by Arzoumanian et al (1996), in which the magnetic inclination angle $\alpha=114^{\circ}$ and the impact parameter $\beta=-19^{\circ}$. If long-term changes in polarization properties can be measured, or if aberration-induced orbital effects can be found, we will have a way to determine the entire orbital geometry of the system, including the vital but currently poorly-constrained spin-orbit misalignment angle, and achieve a test of the rate of precession.

Acknowledgments. We thank Eric Splaver for help with data reduction.

\section{References}

Arzoumanian, Z. 1995, PhD thesis, Princeton University

Arzoumanian, Z. et al. 1996, ApJ, 470, 1111

Cordes, J. M., Wasserman, I. \& Blaskiewicz, M. 1990, ApJ, 349, 546

Kramer, M. 1998, ApJ, 509, 856

Stairs, I. H. et al. 1998, ApJ, 505, 352

Taylor, J. H. \& Weisberg, J. M. 1989, ApJ, 345, 434

Weisberg, J. M., Romani, R. W., \& Taylor, J. H. 1989, ApJ, 347, 1030 